\title{
The concentrations of soluble HLA-G protein are elevated during mid-gestation and decreased in pre-eclampsia
}

\author{
Dorota Darmochwal-Kolarz ${ }^{1}$, Bogdan Kolarz ${ }^{2}$, Jacek Rolinski², \\ Bozena Leszczynska-Gorzelak ${ }^{1}$, Jan Oleszczuk ${ }^{1}$ \\ ${ }^{1}$ Department of Obstetrics and Perinatology, Medical University of Lublin, Poland \\ ${ }^{2}$ Department of Clinical Immunology, Medical University of Lublin, Poland
}

\begin{abstract}
The aim of our study was to investigate the dynamics of the alterations of soluble human leukocyte antigen-G (sHLA-G) concentrations in sera of healthy non-pregnant women, as well as healthy pregnant women and patients with pre-eclampsia. Thirty five patients with pre-eclampsia, 52 healthy pregnant women, and 24 healthy non-pregnant women were included in the study. Sera concentrations of sHLA-G protein were determined using the immunoenzymatic ELISA method. Statistical analysis was performed using ANOVA and Mann-Whitney U tests. The concentrations of sHLA-G protein in sera of pregnant women in the first, as well as the second and third, trimesters of normal pregnancy were significantly higher in comparison with healthy nonpregnant women. The sera concentrations of sHLA-G in pregnant women in the second trimester of pregnancy were significantly higher compared to the first and third trimesters. The concentrations of sHLA-G in sera of patients with pre-eclampsia were significantly lower than in pregnant women in the third trimester of physiological pregnancy. The results of our study suggest that normal physiological pregnancy is associated with elevated sera concentrations of sHLA-G molecule. The increased concentrations of sHLA-G molecule in mid-gestation could suggest a role for the protein in the second phase of a physiological invasion of extravillous cytotrophoblast to spiral arteries. Furthermore, the results could suggest a role for the decreased sera concentrations of sHLA-G in the pathogenesis of pre-eclampsia. (Folia Histochemica et Cytobiologica 2012, Vol. 50, No. 2, 286-291)
\end{abstract}

Key words: pregnancy, pre-eclampsia, sHLA-G

\section{Introduction}

Pre-eclampsia (PE) is a common obstetric syndrome affecting 5-10\% of pregnant women. It is the leading cause of maternal and fetal morbidity and mortality in developed countries. Pre-eclampsia is mostly diagnosed in first pregnancies, and indeed the risk of the disorder in a second pregnancy is significantly reduced when in the first pregnancy normal values of blood pressure have been noted [1,2].

\footnotetext{
Correspondence address: D. Darmochwal-Kolarz, Department of Obstetrics and Perinatology, Medical University of Lublin, Jaczewskiego Str. 8, 20-950 Lublin, Poland; tel.: + 488172447 69, fax: + 488172448 41; e-mail:dorotak@mp.pl
}

Although the symptoms of pre-eclampsia appear in the third trimester of pregnancy, it is generally accepted that the underlying pathologic changes occur much earlier in the placenta and placental bed [3]. It has been proposed that the activation of cell-mediated immunity may play a role in the etiology of preeclampsia. Many alterations in the adaptive immune system have been described which could contribute to the development of this syndrome [4-6]. During the normal course of pregnancy, the process of immunomodulation increases continuously, but it falls in pre-eclampsia [7-9].

Human leukocyte antigen-G (HLA-G) is a nonclassical major histocompatibility complex (MHC) class Ib molecule that is expressed primarily during pregnancy at the maternal-fetal interface. It has multiple immunoregulatory properties. We can distin- 
guish membrane-bound HLA-G and soluble HLA-G (sHLA-G) isoforms. Both isoforms are synthesized by trophoblast cells as well as by fetal cells. The sHLA-G isoform circulates in maternal blood. Its main function in physiological conditions is to protect fetal tissues from the maternal NK cell activity, and to establish immune tolerance at the maternal-fetal interface [10-12]. Alternative splicing of the HLA-G gene results in seven different transcriptional isoforms, of which four encode membrane-bound products, and the other three encode soluble proteins $[10,11]$. The proteins HLA-G1, HLA-G2, HLA-G3 and HLA-G4 are bound to cell surfaces, while HLA-G5, HLA-G6 and HLA-G7 are soluble isoforms [11, 12]. A soluble form of HLA-G protein derived from an alternatively spliced form of mRNA may participate in the vascular remodeling of maternal spiral arteries during pregnancy through the interaction with CD160 [13]. The main isoforms of HLA-G present in serum are sHLA-G1 and sHLA-G5 which are generated by shedding or proteolytic cleavage of the membrane bound isoforms and by secretion of soluble isoforms, respectively. It is abundant in the maternal circulation during pregnancy. They are present not only on cell surfaces, but also in maternal serum, as well as in amniotic fluid. They have also been detected in endothelial cells of fetal vessels in the chorionic villi. Moreover, HLA-G proteins have been found in thymus and fetal liver and on interferon- $\gamma$-stimulated blood monocytes [13]. It remains uncertain whether tolerance to HLA-G develops during fetal life [12]. It has also been shown that the attenuated expressions of HLA-G protein on extravillous trophoblast cells were strongly associated with recurrent pregnancy losses or embryo transfer failures after in vitro fertilization and with pre-eclampsia $[12,16]$. The sHLA-G molecules may be able to block the alloreactive maternal cytotoxic T-cell response via T-cell receptor binding. Therefore, HLA-G protein has a cardinal immune role in several aspects of reproduction including fertility (implantation), gynecology (abortions) and obstetrics (abnormal pregnancies, e.g. PE) $[14,15,18,19]$.

The aim of our study was to investigate the dynamics of the alterations of sHLA-G concentrations in sera of healthy non-pregnant women, as well as healthy pregnant women in the first, second and third trimesters of physiological pregnancy. Furthermore, the aim of the study was to test the hypothesis that sHLA-G concentrations are decreased in sera of patients with pre-eclampsia.

\section{Material and methods}

Patients. The pregnant patients participating in the study were admitted to the Department of Obstetrics and Perina- tology of the Medical University of Lublin. The study population included 35 patients with pregnancy complicated by pre-eclampsia. The diagnosis of pre-eclampsia was made according to the classification criteria of the American College of Obstetricians and Gynecologists. Patients with the following symptoms: elevated blood pressure $>140 / 90 \mathrm{~mm} \mathrm{Hg}$ and with proteinuria $-300 \mathrm{mg}$ or more per 24 hours were qualified as pre-eclamptic and included in the study group. Blood samples from the patients with pre-eclampsia were taken on admission to the hospital before antihypertensive treatment had been given. In most cases, the patients were admitted to the hospital to receive hypertension treatment. Some of them showed intrauterine growth retardation (IUGR). The average blood collection time for pre-eclampsia was $33.02 \pm 3.45$ weeks of gestation. All preeclamptic patients were diagnosed in the third trimester of pregnancy. None of the pre-eclamptic patients was affected by preexisting clinical disorders, such as chronic hypertension, diabetes, overweight/obesity, renal diseases or connective tissue diseases before pregnancy and none of the pregnancies was complicated by preterm labor or chorioamnionitis. The other pregnant patient group comprised 52 healthy women with physiological pregnancy. Blood samples from healthy pregnant women were collected throughout their pregnancies (in the first, second and third trimesters) to minimize patient variance. Blood collection time was as follows: I trimester $-8.47 \pm 1.99$ weeks of gestation; II trimester $-19.37 \pm 2.93$ weeks of gestation; III trimester $-33.41 \pm 3.33$ weeks of gestation. All pregnancies in the study and control groups were singleton. The clinical characteristics of patients with pre-eclampsia and healthy women in the third trimester of uncomplicated pregnancy are given in Table 1.

There were also 24 healthy non-pregnant women with a history of successful pregnancies included in the study. Non-pregnant women (age 19-34) did not take any contraceptive pills. They had regular, biphasic (ovulatory) cycles. Blood samples from non-pregnant women were collected on days $20-23$ of the menstrual cycle. The menstrual cycles of women were monitored. The pre-ovulatory LH surge, the rise in the basal body temperature (BBT), and the changes in cervical mucus were taken into account to assess the ovarian cycle. For non-pregnant women, the pre-ovulatory LH surge was estimated with the use of commercially available ovulation tests measuring the level of $\mathrm{LH}$ in urine samples (Ovulation test, Laboratoire SBH, Strasbourg, France). Complete medical, surgical, and social history was obtained from all women.

Ethical issues. The study design was accepted by the local Ethics Committee. Informed consent from the study subjects for peripheral blood sampling was obtained.

Blood collection. Five milliliters of blood were taken from each patient and every woman from the control group by 
Table 1. Clinical characteristics of patients with pre-eclampsia (study group) and healthy women in the third trimester of uncomplicated pregnancy (control group)

\begin{tabular}{|l|c|c|c|}
\hline & $\begin{array}{c}\text { Study group } \\
\text { Mean } \pm \text { SD } \\
(\mathbf{n}=\mathbf{3 5})\end{array}$ & $\begin{array}{c}\text { Control group } \\
\text { Mean } \pm \text { SD } \\
(\mathbf{n}=\mathbf{5 2})\end{array}$ & $\begin{array}{c}\text { Significance } \\
\text { (p) }\end{array}$ \\
\hline Maternal age & $28.43 \pm 4.67$ & $28.07 \pm 4.83$ & NS \\
\hline Gravidity & $1.79 \pm 1.66$ & $1.72 \pm 0.91$ & NS \\
\hline Parity & $1.56 \pm 0.83$ & $1.39 \pm 0.69$ & NS \\
\hline Time of blood collection (weeks of gestation) & $32.62 \pm 4.17$ & $33.97 \pm 3.48$ & NS \\
\hline Systolic pressure $[\mathrm{mm} \mathrm{Hg]}$ & $152.45 \pm 13.94$ & $113.48 \pm 19.17$ & $<0.01$ \\
\hline Diastolic pressure $[\mathrm{mm} \mathrm{Hg}]$ & $98.34 \pm 4.89$ & $74.77 \pm 6.79$ & $<0.05$ \\
\hline Proteinuria $[\mathrm{g} / 24 \mathrm{hours}]$ & $1.43 \pm 0.85$ & $\mathrm{Absent}$ & $<$ \\
\hline Uric acid $[\mathrm{mg} / \mathrm{dl}]$ & $5.85 \pm 1.43$ & $3.42 \pm 1.25$ & $<0.01$ \\
\hline Creatinine $[\mathrm{mg} / \mathrm{dl}]$ & $1.1 \pm 0.3$ & $0.7 \pm 0.2$ & $<0.05$ \\
\hline Bilirubin $[\mathrm{mg} / \mathrm{dl}]$ & $0.7 \pm 0.3$ & $0.5 \pm 0.2$ & NS \\
\hline Fibrinogen $[\mu \mathrm{g} / \mathrm{l}]$ & $615.35 \pm 120.85$ & $495.05 \pm 0.15$ & NS \\
\hline Fetal weight $[\mathrm{g}]$ & $2,580 \pm 615$ & $3,175 \pm 352$ & $<0.05$ \\
\hline
\end{tabular}

venipuncture in sterile conditions and collected in sterile tubes. Blood samples were centrifuged for $15 \mathrm{~min}$ at $600 \mathrm{~g}$ at $4^{\circ} \mathrm{C}$. Serum was separated from the cells within $15 \mathrm{~min}-$ utes after collection of blood. Next, serum was frozen at $-80^{\circ} \mathrm{C}$ until assayed. Duration of storage before measurement was up to seven months.

The measurements of sera HLA-G concentrations. The concentrations of sHLA-G protein in sera samples were determined by a specific double monoclonal sandwich enzyme immunoassay ELISA technique (BioVendor Laboratory Medicine, Heidelberg, Germany) according to the manufacturer's instructions. It measured shedded HLA-G1 and HLA-G5 isoforms (14). The analytical sensitivity was $1 \mathrm{U} / \mathrm{ml}$. The range of the calibration curve was 3.91-125 Units/ml. The intra-assay coefficient of variation was $5.2-10 \%$. The inter-assay coefficient of variation was $5.5-6.2 \%$. The negative and positive controls used in the study were provided by the manufacturer. Standard and analyzed samples were coated with mouse anti-human sHLA-G monoclonal antibody and put into the microplate wells. The analyzed samples were tested in duplicate. After one-hour incubation at room temperature, sHLA-G protein linked with monoclonal antibody. An antibody overflow was washed out three times with Washing Buffer. Washing Buffer was made from Wash Solution Concentrate. The content of Wash Solution Concentrate is PBS pH 7.4 with detergent $-0.05 \%$ Tween- 20 . Washing Buffer is a solution of $100 \mathrm{ml}$ of Wash Solution Concentrate and $400 \mathrm{ml}$ of distilled water. The next step was to incubate the plate for one hour at room temperature with Conjugate Solution (mouse monoclonal anti-human $\beta 2$-microglobulin antibody, Horseradish Peroxidase Conjugate). The subsequent step was to wash out the plate three times with the Washing Buffer to remove unconnected antibody. Next, the enzyme substrate solution was added and the color reaction was performed. The plate was incubated for 15 minutes. The color intensity depended on the concentrations of sHLA-G molecule in the analyzed samples. Finally, the stop solution $\left(0.2 \mathrm{M} \mathrm{H}_{2} \mathrm{SO}_{4}\right)$ was added and the development of color reaction was inhibited. The absorbance was measured at wavelength $1=450 \mathrm{~nm}$. Standard curve was used to establish the concentrations of sHLA-G protein in analyzed samples.

Statistic analysis. The statistical differences in the group of healthy pregnant women between the first, second and third trimesters of pregnancy were estimated using ANOVA test. The statistical differences between the group of patients with pre-eclampsia and healthy third trimester pregnant women were estimated with the use of Mann-Whitney U test. The results were presented as mean and standard deviation of the data. Differences at $p<0.05$ were considered as statistically significant. Statistica 7.1 (Krakow, Poland) software was applied to statistical analysis.

\section{Results}

The concentrations of sHLA-G molecule in sera of patients with pre-eclampsia were significantly lower than in sera of healthy third trimester pregnant women $(\mathrm{p}<0.01)$.

The concentrations of sHLA-G molecule in sera of healthy first trimester pregnant women were significantly higher than in healthy non-pregnant women $(p<0.005)$. Similarly, the concentrations of sHLA-G in sera of healthy second and third trimester women 
Table 2. Concentrations of sHLA-G molecule (Units/ml) in sera of healthy non-pregnant women, healthy pregnant women in the first, second and third trimesters of physiological pregnancy, and patients with pregnancy complicated by pre-eclampsia

\begin{tabular}{|l|c|c|c|}
\hline & $\begin{array}{c}\text { Number of patients } \\
(\mathbf{n})\end{array}$ & $\begin{array}{c}\text { Mean } \pm \text { SD } \\
{[\mathbf{U} / \mathbf{m l}]}\end{array}$ & $\begin{array}{c}\text { Statistical significance } \\
\text { (p) }\end{array}$ \\
\hline Non-pregnant women & 24 & $10.54 \pm 7.11$ & $\mathrm{p}<0.01$ \\
\hline I trimester women & 52 & $49.14 \pm 31.02$ & $\mathrm{p}<0.001$ \\
\hline II trimester women & 52 & $91.49 \pm 41.58$ & $\mathrm{p}<0.01$ \\
\hline III trimester women & 52 & $63.31 \pm 32.63$ & $\mathrm{p}<0.005$ \\
\hline Patients with pre-eclampsia & 35 & $33.74 \pm 28.30$ & $\mathrm{p}<0.01$ \\
\hline
\end{tabular}

were significantly higher than in healthy non-pregnant women ( $\mathrm{p}<0.0001$ and $\mathrm{p}<0.005$ respectively). Furthermore, the serum concentrations of sHLA-G in pre-eclamptic patients were significantly higher than in healthy non-pregnant women $(\mathrm{p}<0.01)$.

The concentrations of sHLA-G molecule in the sera of women in the second trimester of physiological pregnancy were significantly higher than in the first and third trimesters $(\mathrm{p}<0.01)$. There were no statistically significant differences between the sera concentrations of sHLA-G molecule in the first and the third trimesters of physiological pregnancy. There were negative correlations between the levels of uric acid and the concentrations of sHLA-G in sera of patients with preeclampsia, as well as the levels of creatinine and the concentrations of sHLA-G in sera of patients with preeclampsia $(p<0.05)$. The results of sHLA-G concentrations in sera of patients with pre-eclampsia, as well as healthy pregnant women in the first, second and third trimesters of pregnancy and healthy non-pregnant women, are presented in Table 2.

\section{Discussion}

In pregnancy, the fetus modulates the maternal immune system activity so as not to be rejected from the host organism. It is possible because of the placenta barrier as well as immunomodulatory alterations. It has been suggested that the failure of maternal immunomodulation may be the cause of some complications of pregnancy, e.g. pre-eclampsia, intrauterine growth retardation (IUGR) and placental abruption $[3,4,8]$.

It has been found that sHLA-G molecule is released by endovascular trophoblast cells invading maternal spiral arteries. This could explain its presence in the maternal blood circulation. The antigen HLA-G may play a specific immunosuppressive role during pregnancy $[10,12,14,20,21]$. Although the mechanisms of the immune system modulation by this molecule remain unclear, studies have shown that sHLA-G is necessary for a local tolerance induction at the fetal-maternal interface, transplantation tolerance and probably tumor progression [11, 15, 23-25]. It has also been revealed that mixed lymphocyte cultures, mainly cytotoxic $\mathrm{T}$ lymphocytes, were inhibited by sHLA-G molecule. Moreover, Fournel et al. demonstrated that binding of sHLA-G induces apoptotic $\mathrm{CD}^{+}$ T-cell through the Fas/FasL pathway [27]. It has also been noted that sHLA-G molecules are produced by medullary epithelial thymus cells. This might suggest that central presentation opens the possibility of a thymic HLA-G restricted T cells development [19, 26]. There is a possibility that killing $\mathrm{T}$ cells requires high concentrations of sHLA-G protein that would be expected at the maternal-fetal interface [29, 30]. sHLAG1/G5 has also been identified in supernatant of the embryo's culture as a marker for successful implantation [28].

In our study, we found higher concentrations of sHLA-G molecule in sera of pregnant women in all trimesters of pregnancy compared to healthy non-pregnant women. Furthermore, we observed that the concentrations of sHLA-G molecule increased in the second trimester compared to the first trimester of physiological pregnancy and then decreased in the third trimester of pregnancy compared to the second trimester to levels similar to those in the first trimester of physiological pregnancy. The concentrations of sHLA-G in sera of pregnant women with pre-eclampsia were significantly lower compared to the third trimester of physiological pregnancy.

Yie et al. noticed significantly higher levels of sHLA-G molecule during pregnancy in comparison with non-pregnant women. They observed a decreasing tendency during the normal course of pregnancy. They revealed that the levels of sHLA-G molecule decreased in serum during the third trimester in women who had symptoms of severe PE [28]. Another study showed that sHLA-G concentrations were significantly higher in each of the three trimesters of 
pregnancy than in non-pregnant women [23]. Similarly to our results, Hackmon et al. demonstrated significantly lower levels of sHLA-G molecule during the third trimester of pregnancy in patients suffering from severe pre-eclampsia $[19,21]$. It has also been reported that there is decreased expression of HLA-G antigen in placenta of women with pre-eclampsia compared to normal pregnancies [22].

In the study by Steinborn et al., the levels of sHLA-G1/G5 strongly increased during the first trimester of normal pregnancy compared to non-pregnant women, and then decreased continuously toward term. The pregnant women with significantly decreased sHLA-G1/G5 levels in the second trimester had an increased risk of developing pre-eclampsia and/or IUGR during the further course of pregnancy. However, in the third trimester, sHLA-G1/G5 levels in affected women did not deviate significantly from those of non-affected women. The authors concluded that for the identification of women with an increased risk of IUGR and/or pre-eclampsia, the measurement of sHLA-G1/G5 plasma levels may be a powerful new tool in prenatal diagnostics [21].

It seems possible that more elevated concentrations of sHLA-G in the sera of healthy pregnant women in the second trimester of normal pregnancy may be associated with the disappearance of trophoblast plugs in maternal spiral arteries [30]. On the other hand, the increased risk of pre-eclampsia and/or IUGR in women who had decreased levels of sHLAG1/G5 in the second trimester may be the effect of disturbances in the process of disappearance of trophoblast plugs in the spiral arteries.

The expressions of HLA-G antigen are up-regulated at the time when trophoblast differentiates to an invasive pathway. Furthermore, a soluble form of HLA-G protein derived from an alternatively spliced form of mRNA may participate in the vascular remodeling of maternal spiral arteries during pregnancy through an interaction with CD160. In addition to this, sHLA-G molecule modulates the secretion of cytokines, induces immunotolerance, influences NK cell function, and controls the invasion of trophoblast during pregnancy [30-36]. It is now becoming increasingly clear that it may influence pregnancy outcome at several levels. In normal pregnancy, it appears that the major function of NK cells is to provide benefit by secreting a number of cytokines, chemokines and angiogenic factors rather than to exert a cytotoxic activity [37-39]. It has been found that sHLA-G binds KIR2DL4 receptor which triggers secretion of proangiogenic factors that control vascular uterine remodeling [40, 41].
It is known that during human pregnancy the physiological invasion of cytotrophoblast inside the uterine wall consist of two phases. The first step of the invasion takes place at the beginning of the pregnancy after the process of fertilization. After a long pause, which lasts about 6-8 weeks, there is a second very deep physiological invasion of the cytotrophoblast at the beginning of the second trimester, around 14-16 weeks of gestation. The second invasion of the cytotrophoblast to spiral arteries is completed at 18-20 weeks of gestation $[3,4]$.

The differences between the results of our study and those of other authors could result from different assay forms, different patient group composition, and different time point of collection. The ELISA test used in our study measured sHLA-G1 and HLA-G5 protein concentrations which are the main isoforms presented in serum. The secondary monoclonal antibody used was directed against human $\beta 2$-microglobulin. The use of this antibody excludes the detection of sHLA-G free heavy chains. This test does not discriminate the different sHLA-G isoforms - sHLA-G6 [41]. Furthermore, a large number of patients with preeclampsia and healthy pregnant women were included in our study.

The results of our study suggest that normal physiological pregnancy is associated with elevated sera concentrations of sHLA-G molecule. It seems possible that elevated concentrations of sHLA-G molecule during mid-gestation are associated with the process of disappearance of trophoblast plugs in the spiral arteries during the second phase of a physiological invasion of extravillous cytotrophoblast which is completed at 18-20 weeks of gestation. Furthermore, the results can suggest the role of the decreased sera concentrations of sHLA-G in the pathogenesis of preeclampsia.

\section{Acknowledgments}

This work was supported by grant no N N407 125238 from the Polish Ministry of Sciences.

\section{References}

1. Goodburn E, Cambell O. Reducing maternal mortality in the developing world: sector-wide approaches may be the key. Br Med J. 2001;322:917-920.

2. Hill K, AbouZahr C, Wardlaw T. Estimates of maternal mortality for 1995. Bull World Health Organ. 2001;79:182-193.

3. Norris M, Perico N, Remuzzi G. Mechanism of the disease: pre-eclampsia. Nat Nephrol. 2005;1:98-114.

4. Redman CW. Immunological aspects of pre-eclampsia. Baillieres Clin Obstet Gynaecol. 1992;6:601-615.

5. Darmochwal-Kolarz D, Rolinski J, Tabarkiewicz J, Leszczynska-Gorzelak B, Buczkowski J. Myeloid and lymphoid den- 
dritic cells in normal pregnancy and pre-eclampsia. Clin Exp Immunol. 2003;132:339-344.

6. Darmochwal-Kolarz D, Saito S, Rolinski J, Tabarkiewicz J, Kolarz B, Leszczynska-Gorzelak B. Activated T Lymphocytes in Pre-Eclampsia. Am J Reprod Immunol. 2007;58:39-45.

7. Sacks GP, Studena K, Sargent IL, Redman CWG. Normal pregnancy and pre-eclampsia both pro duce inflammatory chan ges in peripheral blond leukocytes akin to those of sepsis. Am J Obstet Gynecol. 1998;179:80-86.

8. Saito S, Shiozaki A, Nakashima A, Sakai M, Sasaki Y. The role of the immune system in preeclampsia. Mol Aspects Med. 2007;28:192-209.

9. Darmochwal-Kolarz D, Leszczynska-Gorzelak B, Rolinski J, Oleszczuk J. T helper 1- and T helper 2-type cytokine imbalance in pregnant women with pre-eclampsia. Eur J Obstet Gynecol Reprod Biol. 1999 86:165-1670.

10. Carosella ED, Moreau P, Le Maoult J, Le'Discorde M, Dausset J, Rouass-Freiss N. HLA-G molecules: from maternal-fetal tolerance to tissue acceptance. Adv Immunol. 2003;81:199-252.

11. O'Brien M, Dausset J, Carosella ED, Moreau P. Analysis of the role of HLA-G in preeclampsia. Hum Immunol. 2000;61:1126-1131.

12. Markert UR. Immunology of pregnancy. In: Clinical Immunology and Allergy. S. Karger, AG, Basel, Switzerland.

13. Hackmon R, Hallak M, Krup M et al. HLA-G Antigen and Parturition: Maternal Serum, Fetal Serum and Amniotic Fluid Levels during Pregnancy. Fetal Diagn Ther. 2004;19:404-409.

14. Le Bouteiller P, Solier C, Pröll J, Aguerre-Girr M, Fournel S, Lenfant F. Placental HLA-G protein expression in vivo: where and what for? Hum Reprod Update. 1999;5:223-233.

15. Le Discorde M, Moreau P, Rouas-Freiss N, Carosella E. HLA-G: immune tolerance in normal and pathological physiology. Pathol Biol. 2002;50:45-51.

16. Pistoia V, Morandi F, Wang X, Ferrone S. Soluble HLA-G: are they clinically relevant? Semin Cancer Biol. 2007;17:469-479.

17. Hunt JS, Pace JL, Morales PJ, Ober C. Immunogenicity of the soluble isoforms of HLA-G. Mol Hum Reprod. 2003;9:729-735.

18. Colbern GT, Chiang MH, Main EK. Expression of the nonclassic histocompatibility antigen HLA-G by preeclamptic placenta. Am J Obstet Gynecol. 1994;170:1244-1250.

19. Hackmon R, Koifman A, Hyobo H, Glickman H, Sheiner E, Geraghty DE. Reduced third-trimester of soluble human leukocyte antigen $\mathrm{G}$ protein in severe preeclampsia. $\mathrm{Am} \mathrm{J} \mathrm{Ob-}$ stet Gynecol. 2007;197:255.e1-5.

20. Desai N, Filipovits J, Singh P, Beman M, Goldfarb J. Assessment of soluble HLA-G levels in day 3 human embryo culture media using a new ELISA kit: Is there a relationship to embryo quality, development and implantation potential? Fertil Steril. 2005; 84:281-282.

21. Steinborn A, Varkonyi T, Scharf A, Bahlmann F, Klee A, Sohn C. Early detection of decreased soluble HLA-G levels in the maternal circulation predicts the occurrence of preeclampsia and intrauterine growth retardation during further course of pregnancy. Am J Reprod Immunol. 2007;57:277-286.

22. Colbern GT, Chiang MH, Main EK. Expression of the nonclassic histocompatibility antigen HLA-G by preeclamptic placenta. Am J Obstet Gynecol. 1994;170:1244-1250.

23. Alegre E, Díaz-Lagares A, Lemaoult J, López-Moratalla N, Carosella ED, González A. Maternal antigen presenting cells are a source of plasmatic HLA-G during pregnancy:
Longitudinal study during pregnancy. Hum Immunol. 2007;68:661-667.

24. Vianna P, Dalmáz CA, Veit TD, Tedoldi C, Roisenberg I, Chies JA. Immunogenetics of pregnancy: Role of a 14-bp deletion in the maternal HLA-G gene in primiparous preeclamptic Brazilian women. Hum Immunol. 2007;68:668-674.

25. Zavazava N, Krönke M. Soluble HLA class I molecules induce apoptosis in alloreactive cytotoxic T lymphocytes. Nat Med. 1996;2:1005-1010.

26. Hunt JS, Petroff MG, Morales P, Sedlmayr P, Geraghty DE, Ober C. HLA-G in reproduction: studies on the maternal-fetal interface. Hum Immunol. 2000;61:1113-1117.

27. Fournel S, Aguerre-Girr M, Huc X et al. Soluble HLA-G1 triggers CD95/CD95 ligand-mediated apoptosis in activated CD8+ cells by interacting with CD8. J Immunol. 2000;164: 6100-6104.

28. Yie SM, Li LH, Li YM, Librach C. HLA-G protein concentrations in maternal serum and placental tissue are decreased in preeclampsia. Am J Obstet Gynecol. 2004;191:525-529.

29. Noci I, Fuzzi B, Rizzo R et al. Embryonic soluble HLA-G as a marker of developmental potential in embryos. Hum Reprod. 2005;20:138-146.

30. Yie SM, Taylor RN, Librach C. Low plasma HLA-G protein concentrations in early gestation indicate the development of preeclampsia later in pregnancy. Am J Obstet Gynecol. 2005;193:204-208.

31. Maejima M, Fujii T, Kozuma S, Okai T, Shibata Y, Taketani Y. Presence of HLA-G-expressing cells modulates the ability of peripheral blood mononuclear cells to release cytokines. Am J Reprod Immunol. 1997;38:79-82.

32. Rizzo R, Andersen AS, Lassen MR et al. Soluble human leukocyte antigen-G isoforms in maternal plasma in early and late pregnancy. Am J Reprod Immunol. 2009;62:320-338.

33. Menezo Y, Elder K, Viville S. Soluble HLA-G release by the human embryo: an interesting artifact. Reprod Biomed Online. 2006;13:763-764.

34. LeRond S Le Maoult J, Creput $\mathrm{C}$ et al. Alloreactive $\mathrm{CD}^{+}$ and $\mathrm{CD} 8^{+} \mathrm{T}$ cells express the immunotolerant HLA-G molecule in mixed lymphocyte reactions: in vivo implications in transplanted patients. Eur J Immunol. 2004;34:649-660.

35. Chaouat G. Current knowledge on natural killer cells, pregnancy and pre-eclampsia. Introduction. Reprod Biomed Online. 2008;16:170-172.

36. Le Bouteiller P. The role of HLA-G expression in the embryo during implantation. J Gynecol Obstet Biol Reprod. 2004;33:9-12.

37. Le Bouteiller P, Pizzato N, Barakonyi A, Solier C. HLA-G, pre-eclampsia, immunity and vascular events. J Reprod Immunol. 2003;59:219-234.

38. Santoni A, Carlino C, Stabile H, Gismondi A. Mechanisms underlying recruitment and accumulation of decidual NK cells in uterus during pregnancy. Am J Reprod Immunol. 2008;59:417-424.

39. Santoni A, Carlino C, Gismondi A. Uterine NK cell development, migration and function. Reprod Biomed Online. 2008; 16:202-210.

40. Rajagopalan S, Long EO. Antagonizing inhibition gets NK cells going. Proc Natl Acad Sci USA. 2010;107:10333-10334.

41. Hunt JS, Morales PJ, Pace JL, Fazleabas AT, Langat DK. A commentary on gestational programming and functions of HLA-G in pregnancy. Placenta. 2007;28:57-63.

Submitted: 20 February, 2011

Accepted after reviews: 8 January, 2012 